\title{
Verhandlungen der dermatologischen Gesellschaft zu Stockholm.
}

\author{
Sitzung rom 22. September 1910 .
}

Schlasberg demonstriert Fälle von Lichen syphiliticus, von Lichen planus universalis und von Lichen planus des Gesichtes, der Handrücken und der Schleimhaut der Unterlippe.

Almkvist. 8jähr. Knabe mit einem Riesennaevus des Rückens; Länge $28 \mathrm{~cm}$, größte Breite $19 \mathrm{~cm}$; reichlicher Haarwuchs und braun pigmentierte, stellenweise hyperkeratotische Haut.

Afzelius. 30jähr. Mann; seit 2-3 Jabren chronisches diffuses Ekzem der Unterschenkel und seit einigen Monaten lineäres des rechten Oberschenkels, an dessen Innenseite sich ein $2-3 \mathrm{~mm}$ breites Band von juckenden, für Lichen planus nicht typischen, teilweise schuppenden Papeln findet, das von der Kniebeuge bis zur Leistenbeuge sich erstreckt; vielleicht entspricht dieser Verlauf einigen Lymphbahnen dieser Gegend.

Moberg. Ein Fall von ausgebreitetem Lichen planus mit arthritischen Symptomen und ein älterer Mann mit Sarcoma idiopathicum haemorrbagicum multiplex; der Patient wurde vor zwei Jahren in dieser Gesellschaft vorgestellt, ist seitdem mit Röntgen behandelt worden und es ist jetzt sehr wenig von seiner Krankheit zu sehen.

Schauman. 43jähr. Mann mit multiplen, follikularen Zysten. Vor 3 Jahren kleine, indolente Knötchen, von der Farbe der umgebenden IIaut, an der Hinterseite des Halses; später ähnliche im okzipitalen Teile des Kapillitium; seit einem Jahre auch an der Stirne. Stat. praes.: Zahlreiche solche Knötchen mit angegebener Lokalisation. Im hinteren Teile des Kapillitium, zwischen den Knötchen, zahlreiche kleine Alopezien. Keine Komedonen, Akne oder erweiterte Follikelmündungen. Mikroskopische Untersuchung: Mit Hornlamellen und Haaren ausgefüllte Retentionszysten, durch Dilatation der für 2-4 Follikeln gemeinsamen Infundibula (aperturae communes) entstanden; die Ursache dieser Stagnation ist vielleicht in der eigenartigen Konfiguration der gemeinsamen Aperturen (ungewöhnlich tief oder deformiert) zu suchen. Auf Grund der Stagnation entstehen sekundäre Veränderungen in 1. der Par s media et inferior der Follikeln, die oft durch Atrophie in solide Zäpfchen am Fundus der Zyste reduziert sind und 2. den Talgdrü sen, welche im allgemeinen atrophisch (kleine zapfenförmige Anhängsel) oder auch vollständig verschwunden sind. Oft ist auch eine zystische Dilatation des Ausführungsganges und hie und da Erweichungszysten im Talgdrüsenparenchyme (bisweilen die ganzen Talgdrüsenkörper ausfüllend) zu sehen. Der Prozeß resultiert im Zerfall der betreffenden Follikeln und Resorption durch ein riesenzellreiches Granulationsgewebe.

Strandberg. Drei Fälle von $P$ blebitis syphilitica mit Demonstration mikroskopischer Präparate.

Über ihre bisherigen Erfahrungen mit Ehrlichs Dioxidiamidoarsenobenzol bei Syphilis geben Strandberg. Schanman, K. Marcus, Almkvist, H. Marcus und Gron (aus Christiania) vorläufige Mitteilungen; nach einer genügenden Beobachtungszeit der behandelten Fälle (zusammen ungefähr 100) werden die Ausführungen von den betreffenden Klinikvorständen veröffentlicht werden.

Karl Marcus (Stockholm). 